\title{
Procedural Function-based Modelling of Volumetric Microstructures
}

\author{
Alexander Pasko $^{1}$, Oleg Fryazinov ${ }^{1}$, Turlif Vilbrandt ${ }^{2}$, Pierre-Alain Fayolle ${ }^{3}$, Valery Adzhiev ${ }^{1}$ \\ ${ }^{1}$ Bournemouth University, UK \\ ${ }^{2}$ Digital Materialization Group, Japan, and Uformia AS, Norway \\ ${ }^{3}$ University of Aizu, Japan
}

\begin{abstract}
We propose a new approach to modelling heterogeneous objects containing internal volumetric structures with size of details orders of magnitude smaller than the overall size of the object. The proposed function-based procedural representation provides compact, precise, and arbitrarily parametrised models of coherent microstructures, which can undergo blending, deformations, and other geometric operations, and can be directly rendered and fabricated without generating any auxiliary representations (such as polygonal meshes and voxel arrays). In particular, modelling of regular lattices and cellular microstructures as well as irregular porous media is discussed and illustrated. We also present a method to estimate parameters of the given model by fitting it to microstructure data obtained with magnetic resonance imaging and other measurements of natural and artificial objects. Examples of rendering and digital fabrication of microstructure models are presented.
\end{abstract}

Keywords: microstructure, lattice, porous media, tissue engineering, scaffold, function representation, digital fabrication

\section{Introduction}

Recent developments in computing allow researchers to look beyond traditional geometric modelling techniques oriented towards surface based and homogeneous solid models. Man-made objects are often nearly uniform in their internal structure, for example, most of mechanical parts can be modelled as homogeneous solids. In contrast, natural objects are rarely homogeneous having a complicated internal structure and materials distribution.

New application areas of CAD/CAM such as biomedical tissue engineering [1] and composite materials in mechanical engineering [2] deal with essentially heterogeneous objects. Such objects can be made of different materials with variable densities and can have internal microstructures. The common feature of such objects is presence of internal volumetric structures with size of details orders of magnitude smaller than the overall size of the object. These can be lattices, porous, branching or granular material compositions varying from regular to completely irregular random structures. Not only are microstructures important in engineering new complex objects, but are becoming increasingly important for manufacturing traditional objects using digital fab-

Preprint submitted to Graphical Models rication processes. Adding internal microstructures to objects reduces weight, materials, energy and time required to create an object. In the future it is likely that most large, digitally fabricated objects will not be created as solid blocks of materials but will instead be made up of microstructures.

Existing approaches to modelling microstructures include those relying on surfaces (boundary representations or BRep) and voxels (discrete volume representations). Although to some extent microstructures can be generated using these representations (see the next section), many of the known problems and limitations of both representations are amplified by the geometric complexity of microstructures. The problem compounds further when modelling irregular microstructures. One can distinguish between quantitative problems (model size and processing time) and qualitative problems (model validity, precision, parameterization, operability, and manufacturability) of existing representations:

- Size and processing time

Surface based models of a moderate size containing high quality lattice microstructures can include such a huge number of polygons that it becomes

February 18, 2011 
difficult or impossible for modern graphics hardware to render. Although the size of voxel models is not directly dependent on the object complexity, the number of voxels needed to represent high quality surfaces along with small details can easily exceed available capacities of computer memory. Time for rendering as well as for other types of processing grows with the size of the model.

\section{- Validity and precision}

Known problems arising from traditional BRep based CAD models and approaches (such as cracks in surfaces, self-intersections of polygons, additional false polygons left over from modelling, and inverted normal orientation) already problematic for the manufacturing industry become increasingly difficult or impossible to model and manufacture at nano- and micro- scales. BRep and voxel models are usually created inside some specifically given bounding unit and then iteratively replicated in space without considering spatial coherence of the structure, which can cause additional cracks in the model at the boundaries of each unit. Likewise, the "skinning" or proper connection of a microstructure to the object shell presents many unsolved issues for current systems. In general, both the polygonal BRep and the voxel representation are not exact and only approximate the modelled geometry with limited precision.

\section{- Parameterization and operability}

The support of model generation with variable parameters is crucial for modelling microstructures, because their geometry can depend on a number of factors such as distance to the object surface, predefined strength conditions, density variations, and others. The existing representations have limited or no support for parameterization of microstructure models. When parameters are changed, BRep and voxel models have to be re-generated using a separate, high level procedure or method. A user may need to apply further specific operations on microstructures such as offsets, blends and shape deformations, which have limited or no support within the current representations.

\section{- Manufacturability}

Controlled complex microstructures are not easy to manufacture using most existing technologies. Currently the best solution can be found in rapid prototyping or digital fabrication technologies such as laser sintering, stereolithography and other additive processes, which produce a physical object layer by layer [3][4][1]. As object becomes large or finely packed microstructures are required, BRep models dramatically increase in size and become very hard or impossible for current hardware systems to visualize and even more difficult to cross-section as is required by many digital fabrication systems and processes. While current digital fabrication systems have limited resolutions, in recent years they have achieved ever greater accuracy and this trend is expected to continue. Even so, it is currently not uncommon to see defects or missing sections of an object, largely due to the complexity involved in creating proper crosssections from the standard STL format. Some but not all digital fabrication systems accept voxel models as input, however voxels have known aliasing problems unless they are given at very high resolutions requiring large amounts of memory. For systems that do not work with voxels, polygonization of the voxel data is necessary.

The objective of this work is to develop an approach to the modelling and manufacturing of microstructures that resolves most of the above issues. We propose a compact, precise, and arbitrarily parametrised constructive model based on real functions of point coordinates allowing for the procedural generation of spatially coherent microstructures, which can undergo blending, offsetting, deformations, and other geometric operations. Such models can be automatically fitted to scan data of real microstructures, and can be directly rendered and manufactured without generating any auxiliary representations.

In this work, we concentrate on modelling regular microstructures such as lattices and cellular structures, as well as irregular microstructures such as porous media. By "lattice" we mean a periodic spatial structure consisting of crossing rods, laths or other thin strips of material. We use the term "cellular structures" to designate the periodic replication of the given geometry inside some unit cell within a bounding box of known size and location. The term "porous media" describes various materials that exhibit the property of irregular porosity through an interconnected network of cavities (pores) within a solid material stratum. We try to avoid using the term "scaffold" because it can be applied to both types of structures.

In the next section we outline the prior work on all the above mentioned types of microstructures as well as the background of the Function Representation (FRep) in geometric modelling. Sections 3 and 4 respectively 
present the proposed approaches to modelling regular microstructures (lattices and cellular structures) and irregular structures (porous media). Fitting of parameterized template models to scan data is presented in Section 5 . Section 6 briefly outlines the approaches to direct rendering and direct fabrication avoiding auxiliary representations. The paper is concluded with some discussion of open problems in the last Section 7.

\section{Prior work}

In this section we first describe the main approaches to modelling microstructures and then introduce the basics of the Function Representation for geometric models.

\subsection{Regular microstructures}

Generation of a polygonal model of a regular lattice with cubic elements for visualization is quite trivial using standard surface primitives such as cylinders or blocks. The problem becomes more complex if a valid solid (BRep) model is required for further geometric operations (such as blending between the lattice rods and the outer surface) or digital fabrication. Existing publications provide quite limited information on procedures for the generation of lattice models and primarily concentrate on lattices for specific applications.

A geometric modelling method for creating conformal lattice structures was proposed in [5]. Regular cellular structures are built as a set of unit trusses (cells). The solid model of each unit truss is created using Boolean operations performed in a commercial solid modelling system. Copies of the unit truss are then directly stacked together to generate the model of the entire cellular structure. However, it was pointed out that it took significant computational resources to generate the models of lattice structures since the generation of each unit truss required several Boolean operations. This work was extended in [6] to more general regular internal structure design with truss geometry given as a mesh prior to its replication. The proposed 3D texture mapping approach is based on mapping of a 3D microstructure pattern (truss geometry) into a design space to generate internal cellular structures which then are combined with a given CAD model of the object.

A classification of regular microstructures is given in [1] along with the outline of the modelling process in biomedical applications involving predefined microstructure patterns and scanned biological shapes such as human bones. The typical steps of lattice design in biological tissue engineering and fabrication are described in [4]. A commercial BRep solid modelling system is used to generate lattices for bone implants with the selected spatial structure parametrised by pore sizes, porosity, and surface area to volume ratio. The overall required shape of the model is taken from human body data collected by magnetic resonance imaging. The Boolean intersection is applied to produce a lattice within the patient's bone shape.

Optimization of lattices is presented in [7][8] using maximal strength, minimal weight, and other criteria. The lattice structure (namely, connections between lattice nodes and positions of nodes) is optimized to satisfy the selected criteria.

\subsection{Irregular microstructures}

Modelling irregular microstructures such as porous media is much more complex than modelling regular microstructures because of irregularities essential to pores' locations and shapes. It can be stated that available CAD methods and tools are not adequate for solving this problem. There are two main approaches pursued in recent works: image-based reconstruction [1][9][10] of a surface and voxel models, and pseudorandom simulation [11][12][13].

The process of obtaining geometric surface models for biological porous tissues is described in [1]. It includes obtaining 2D images of the internal tissue structure using computer tomography and other techniques; reconstruction of a 3D voxel model from a set of images; and construction of a surface model through the tessellation of a selected isosurface or by the voxel model segmentation and fitting with predefined parameterized surface primitives.

To reconstruct a $3 \mathrm{D}$ model of a porous structure, the authors of [9] construct a voxel model from a set of cross-section images of a biological material sample. The voxel model is segmented into three regions: exterior, material and pores. A graph is built reflecting individual pores and connections between them. A geometric model can be generated and visualized where each pore is represented by a sphere with the estimated radius.

A process for customised modelling of fitting microimplants into bones based on a volumetric texture synthesis is described in [10]. First, a 3D model is reconstructed from digitized slice-by-slice 2D images along with extracting microstructures from 2D/3D images. Then volumes in the mesh which represent "unhealthy" holes in the bone structure (characterised by sparse and relatively thin trabeculae) are identified. Each 3D hole is in-filled using irregular samples taken from a matching "healthy" region located around the hole. The Ex- 
tended Voxel-by-Voxel (EVolV) texture synthesis algorithm allowing mapping a matching block of voxels rather than only one voxel has been proposed for that purpose.

A direct approach to 3D modelling of porous media is presented in [11]. First, a number of microspheres, which can be packed within the given volume, is estimated. Then the selected number of microspheres is randomly packed in the volume. The Boolean subtraction of the microspheres model from the basic volume model using a commercial BRep modeller results in the porous volume structure. The experiments were conducted with a quite limited number of microspheres (about 50).

A model of a porous object is represented in [12] as a Constructive Solid Geometry (CSG) tree with stochastically generated primitives (spheres) representing pores in the leaves of the tree structure and regularized Boolean operations placed in its nodes. The structure analysis is performed on the voxelized version of this model. A 3D porous structure can be built as a stack of thin extruded 2D slices. The structure in each slice is modelled in [13] using a Voronoi diagram built for the structure generating points moving on the plane.

We can conclude that BRep and voxels remain the main models researchers use to represent microstructures. The major drawbacks of these representations were discussed in the previous section. In the remainder of the paper we tackle these problems using procedural function-based models in the form of the Function Representation (FRep).

\subsection{FRep basics}

The main idea of the function-based modelling (or "implicit surface" modelling [14]) is to define point sets via trivariate scalar fields or continuous real functions of point coordinates $\mathrm{F}(\mathrm{x}, \mathrm{y}, \mathrm{z})$. The point membership depends on the sign of the defining function evaluated at that point, typically points with positive and zero function values are included in the set, while zero-level subset is considered a boundary. This approach to geometric modelling was extended in the Function Representation (FRep) [15] by explicitly introducing elementary objects (primitives) and operations on using the corresponding operations on defining functions.

One particular class of the FRep operations are settheoretic ones defined by R-functions [16][17][15]. An object resulting from the set-theoretic operations has the defining function expressed as follows:

$$
\begin{array}{ll}
f_{3}=f_{1} \vee_{\alpha} f_{2} & \text { for the union; } \\
f_{3}=f_{1} \wedge_{\alpha} f_{2} & \text { for the intersection; } \\
f_{3}=f_{1} \backslash_{\alpha} f_{2} \quad \text { for the subtraction; }
\end{array}
$$

where $f_{1}$ and $f_{2}$ are defining functions of initial objects and $\vee_{\alpha}, \wedge_{\alpha}, \backslash_{\alpha}$ are signs of R-functions. One of the classes of R-functions is

$$
\begin{aligned}
& f_{1} \vee_{1} f_{2}=\max \left(f_{1}, f_{2}\right) \\
& f_{1} \wedge_{1} f_{2}=\min \left(f_{1}, f_{2}\right) \\
& f_{1} \backslash_{1} f_{2}=\min \left(f_{1},-f_{2}\right)
\end{aligned}
$$

These functions are $C^{1}$ discontinuous at all points where $f_{1}=f_{2}$. R-functions of another class:

$$
\begin{aligned}
& f_{1} \vee_{0} f_{2}=f_{1}+f_{2}+\sqrt{f_{1}^{2}+f_{2}^{2}} \\
& f_{1} \wedge_{0} f_{2}=f_{1}+f_{2}-\sqrt{f_{1}^{2}+f_{2}^{2}} \\
& f_{1} \searrow_{0} f_{2}=f_{1}-f_{2}-\sqrt{f_{1}^{2}+f_{2}^{2}}
\end{aligned}
$$

have $C^{1}$ discontinuity only at the points where both arguments are equal to zero.

Implicit surfaces have been recently applied to modelling the internal structure of a biological cell [18] including thousands of irregularly shaped components on the micro-level. Stolte [19] presented replication of implicit surfaces in space using the triangle wave function. Shapiro [17] showed that a periodic regular structure such as a chess board can be modelled using trigonometric functions defining horizontal and vertical strips, which then are combined using the set-theoretic union. We further develop the logical construction based modeling of microstructures. Our experimental models are implemented in the HyperFun language [20], which fully supports the FRep modelling paradigm.

\section{Modelling regular microstructures}

To overcome the known problems of the BRep and voxel based models, we propose to describe microstructures using real functions of point coordinates combined in a constructive manner following the FRep approach presented above. The main requirement for a defining function is to have at least $C^{0}$ continuity. FRep models of microstructures are very compact, precise, and arbitrarily parameterized. They can undergo geometric transformations typical for FRep models: set-theoretic operations, blending, deformations, and many others. In this section, we model infinite regular lattices using 
trigonometric periodic functions and R-functions. For infinite cellular structures with general unit cell geometry, we apply a periodic space mapping while maintaining the defining function continuity.

\subsection{Modelling lattice microstructures}

We propose the following approach to modelling infinite regular 3D lattices:

1. a set of infinite parallel slabs orthogonal to each coordinate axis can be defined by a corresponding periodic function;

2. the intersection of two of these sets results in the infinite rods parallel to one of the axes;

3. the union of rods gives us an infinite rectangular lattice.

To define the infinite slabs, the following functions can be applied:

$$
\begin{aligned}
& s_{x}(x, y, z)=\sin \left(q_{x} x+p_{x}\right)-l_{x} \\
& s_{y}(x, y, z)=\sin \left(q_{y} y+p_{y}\right)-l_{y} \\
& s_{z}(x, y, z)=\sin \left(q_{z} z+p_{z}\right)-l_{z}
\end{aligned}
$$

where the inequality $s_{x} \geq 0$ describes a set of slabs orthogonal to $\mathrm{x}$-axis and parallel to each other, the frequency $q_{x}$ defines the distance between parallel slabs along $\mathrm{x}$-axis, the phase $p_{x}$ defines the position of slabs on the $x$-axis relative to the origin, and the threshold $-1<l_{x}<1$ together with the frequency defines the thickness of each slab. The slabs orthogonal to $y$ and $z$ axes are symmetrically defined by the functions $s_{y}$ and $s_{z}$.

The next step is to describe three sets of rods parallel to each axis:

$$
\begin{aligned}
& r_{x}(x, y, z)=s_{y} \wedge_{\alpha} s_{z} \\
& r_{y}(x, y, z)=s_{x} \wedge_{\alpha} s_{z} \\
& r_{z}(x, y, z)=s_{x} \wedge_{\alpha} s_{y}
\end{aligned}
$$

Here the inequality $r_{x} \geq 0$ describes a set of rods parallel to $x$-axis and obtained as the set-theoretic intersection between slabs orthogonal to $y$-axis and $z$-axis using an R-function (Eqs. 1-3). The final infinite regular lattice is a union of all the rods and can be described as follows:

$$
\begin{aligned}
& g(x, y, z)=r_{x} \vee_{\alpha} r_{y} \vee_{\alpha} r_{z} \\
& \text { or } \\
& g(x, y, z)=\left(s_{y} \wedge_{\alpha} s_{z}\right) \vee_{\alpha}\left(s_{x} \wedge_{\alpha} s_{z}\right) \vee_{\alpha}\left(s_{x} \wedge_{\alpha} s_{y}\right)
\end{aligned}
$$

According to the properties of R-functions, the above function with $\alpha=0$ for the regular lattice is $C^{1}$ continuous in the entire domain, except its surface edges resulting from the intersection and union operations. Note that all the parameters in the above lattice model can be made variable. Fig. 1 illustrates the construction of the regular lattice with some constant parameters (Fig. 1 a,b) and with the rod thickness controlled by the thresholds linearly changing along the horizontal axis (Fig. 1c).

The rods have square in cross-section. Other types of cross-sections can be obtained by using affine transformations over the slabs. To obtain ellipse or circle in cross-section, offsetting operation can be used. In this case in 4 we set $l=1$ and apply offset:

$$
\begin{aligned}
& s_{x}(x, y, z)=\sin \left(q_{x} x+p_{x}\right)-1 \\
& s_{y}(x, y, z)=\sin \left(q_{y} y+p_{y}\right)-1 \\
& s_{z}(x, y, z)=\sin \left(q_{z} z+p_{z}\right)-1 \\
& r_{x}(x, y, z)=s_{y} \wedge_{\alpha} s_{z}+d_{x} \\
& r_{y}(x, y, z)=s_{x} \wedge_{\alpha} s_{z}+d_{y} \\
& r_{z}(x, y, z)=s_{x} \wedge_{\alpha} s_{y}+d_{z}
\end{aligned}
$$

In case $d_{x} \equiv d_{y} \equiv d_{z}$ we obtain circle in cross-section for all the rods, otherwise we obtain ellipse. Fig. 2 illustrates the construction of the regular lattice with circle in cross-section.

All operations that can be applied to functionallybased models can be applied to lattices. For example, we can obtain cylindrical lattices by using mapping to cylindrical coordinates (Fig. 3a). Note that this mapping is a non-linear transformation, and because of that the width of the lattices increases with increasing of the distance from the centre of the cylinder. By applying the tapering operation, we can vary a frequency of the lattices in the selected area or globally (Fig. 3b). Also we can modify the linear nature of lattices by applying the twist operation (Fig. 3c).

The controlled blending versions of set-theoretic operations are applicable to FRep objects [15]. Instead of producing sharp edges, these operations result in smooth transitions between two given surfaces while a set operation is applied to two solids. The formulation of a blending operation is based on the displacement added to a standard R-function, for example for the blending intersection, we have:

$$
f_{1} \wedge_{b} f_{2}=\left(f_{1} \wedge_{\alpha} f_{2}\right)+\frac{a_{0}}{1+\left(\frac{f_{1}}{a_{1}}\right)^{2}+\left(\frac{f_{2}}{a_{2}}\right)^{2}}
$$

where $\wedge_{\alpha}$ stands for one of the R-functions defining the intersection (Eq. 3) and the additional term defines the 


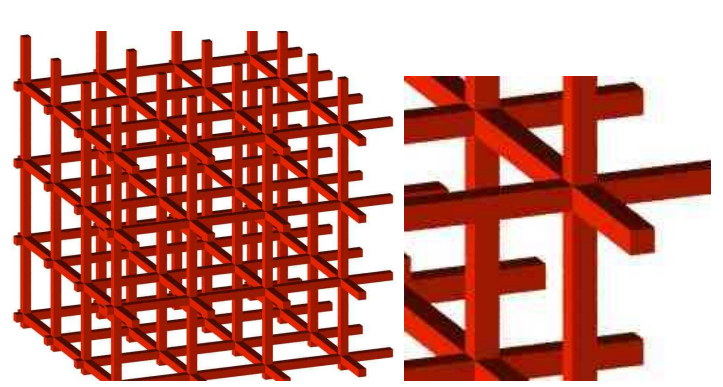

(a)

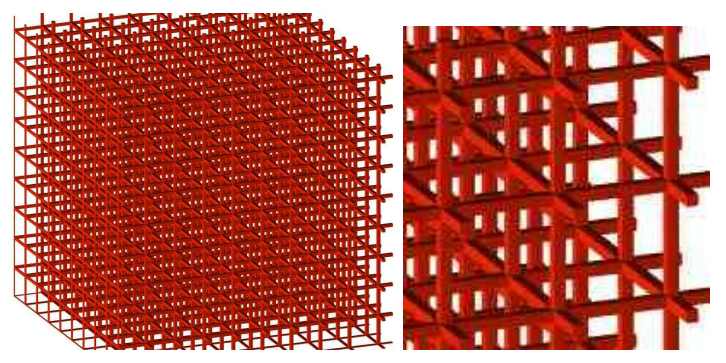

(b)

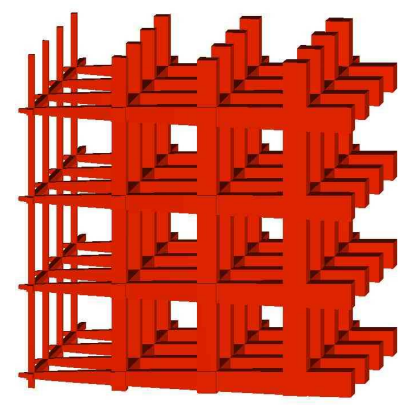

(c)

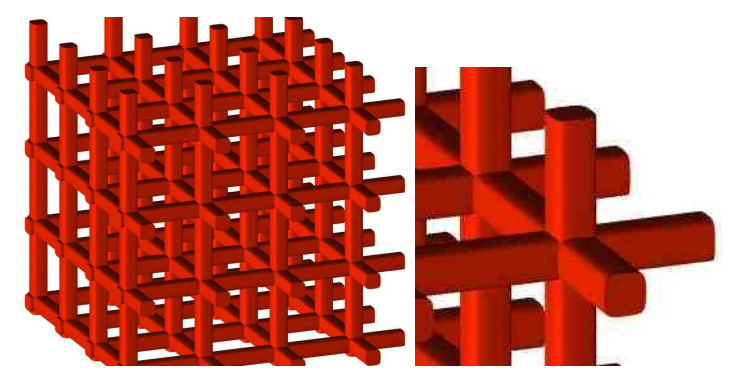

(d)

Figure 1: Function-based infinite regular lattice with (a) constant parameters; (b) triple frequency (c) variable parameters, the rod thickness grows linearly along one axis; (d) rods smoothed by blending. Zooming is provided for images (a), (b) and (d).

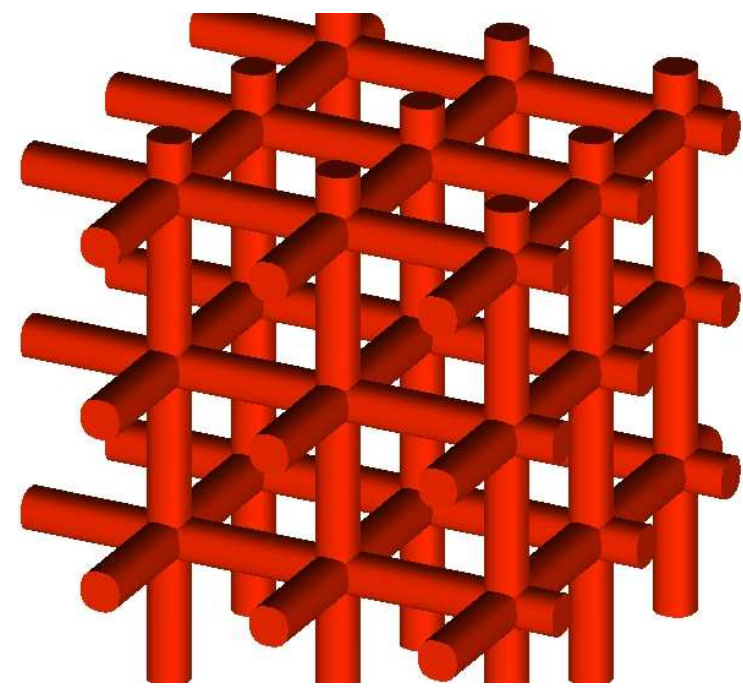

(a)

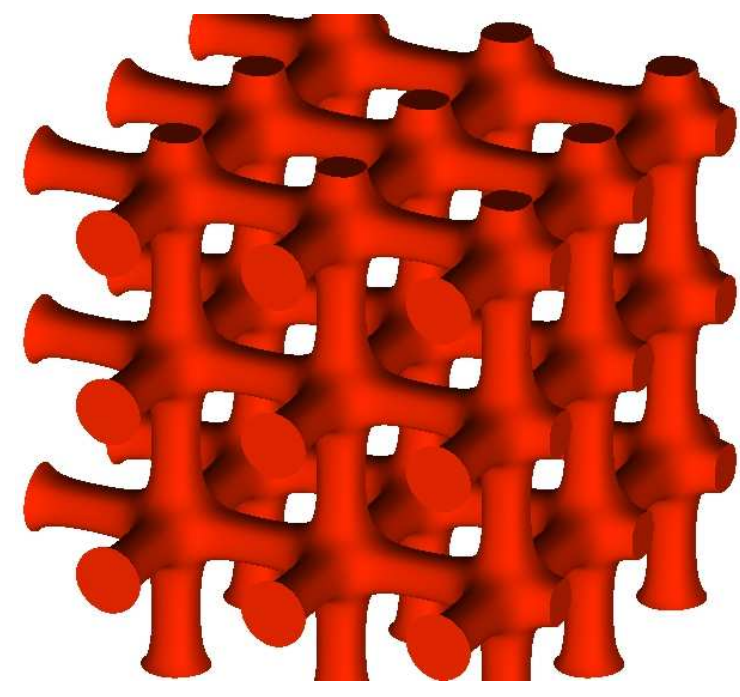

(b)

Figure 2: Construction of cylindrical rods: a) Union of basic cylindrical rods forming a lattice b) Applying blending union between rods instead of the pure union 


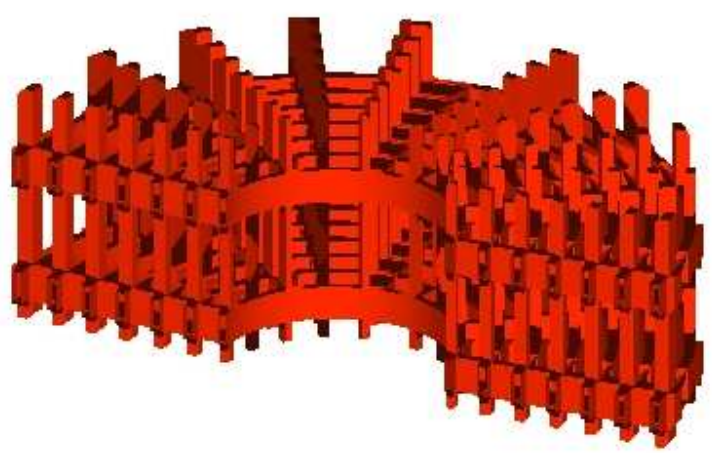

(a)

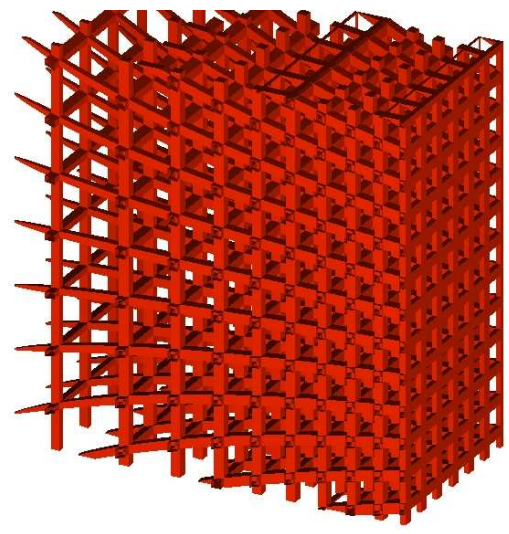

(b)

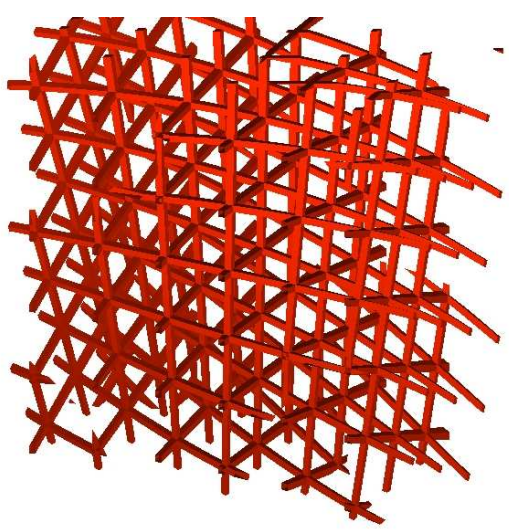

(c)

Figure 3: Applying transformation to function-based lattices: a) Cylindrical mapping b) Tapering c) Twisting displacement with the parameters $a_{0}, a_{1}, a_{2}$ controlling the shape of the blend. For example, $a_{0}<0$ corresponds to a blend with removed material (chamfer). Fig. 1d illustrates an application of this operation to the rods construction, where the intersection operations of Eq. 5 are replaced by the blending intersection (Eq. 8). Similarly, blending union operations can be applied to the rods forming the lattice as shown in Fig. $2 \mathrm{~b}$.

It is important for most of applications to be able to combine a microstructure with the given external shape of the object. Typically a thin shell of the object is created and the microstructure is truncated to fit inside the shell. These operations for the function $F$ defining the initial external shape can be described as follows:

$$
F_{o}(x, y, z)=F(x, y, z)-l_{o}
$$

This function defines the internal offset of the given shape, where $l_{o}>0$ is the offset threshold. The shell of the object is defined as the subtraction of the offset from the initial shape:

$$
F_{s}(x, y, z)=F \backslash_{\alpha} F_{o}
$$

The constant thickness of the thin shell can be achieved with this operation for defining distance functions or with normalized functions approximating the distance function near the surface [21].

The finite lattice within the object can be obtained by intersecting the infinite lattice with the given object:

$$
F_{g}=g \wedge_{\alpha} F
$$

and finally the object shell with the microstructure inside has the defining function

$$
F_{m}(x, y, z)=F_{s} \vee_{\alpha} F_{g}=\left(F \backslash_{\alpha} F_{o}\right) \vee_{\alpha}\left(g \wedge_{\alpha} F\right)
$$

The above construction is illustrated by Fig. 4a, where the function $F$ defines a sphere. If the union operation in Eq. 9 is replaced by a blending union operation, the microstructure rods will be blended with the shell as shown in Fig. 4 b. More complex shapes can undergo similar operations. Fig. 5 illustrates a practical example of the lattice scaffold design for a jaw bone. The given shape in this case is an FRep model fitted to a scanned bone.

The lattice parametrization resulting in variable density and rod sizes can be made dependent not only on spatial coordinates, but on other factors. In biomedical engineering the lattice scaffolds have to be denser near the surface. This can be achieved by making lattice parameters dependable either on the distance to the surface or, in the case of FRep object, on the defining function, which takes zero value on the boundary (see Fig. 4c). 


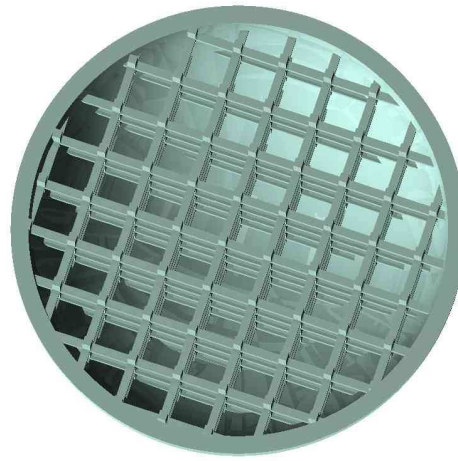

(a)
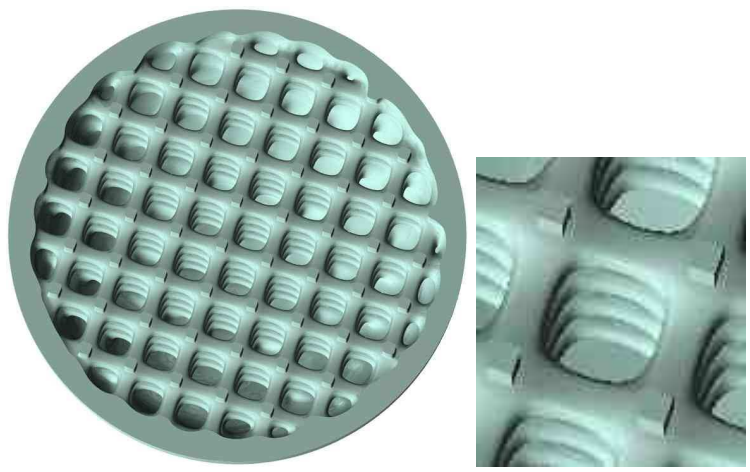

(b)

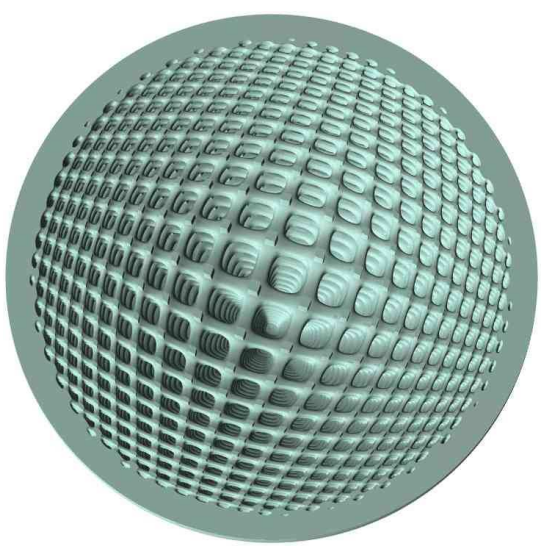

(c)

Figure 4: Combining microstructures with external shapes: (a) union of a spherical shell with a regular lattice; (b) blending union between the rods of the regular lattice, and between the rods and the spherical shell; (c) lattice parametrization depending on the distance to the external surface.

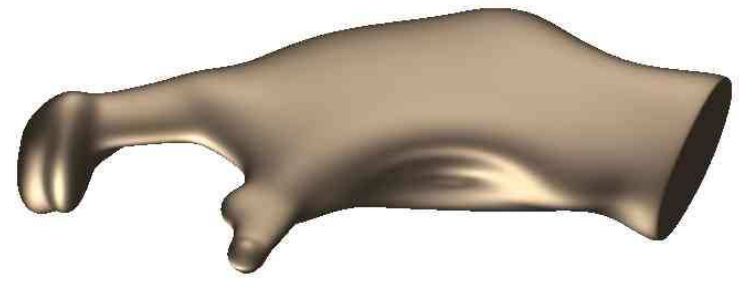

(a)

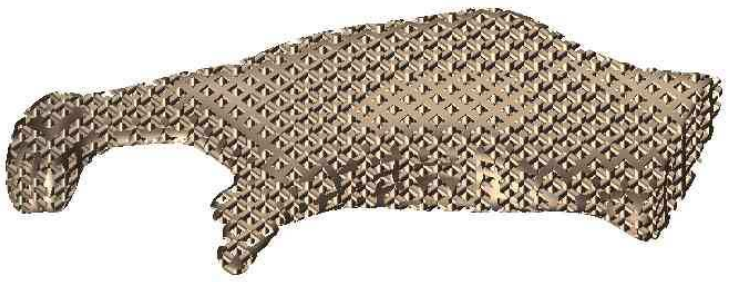

(b)

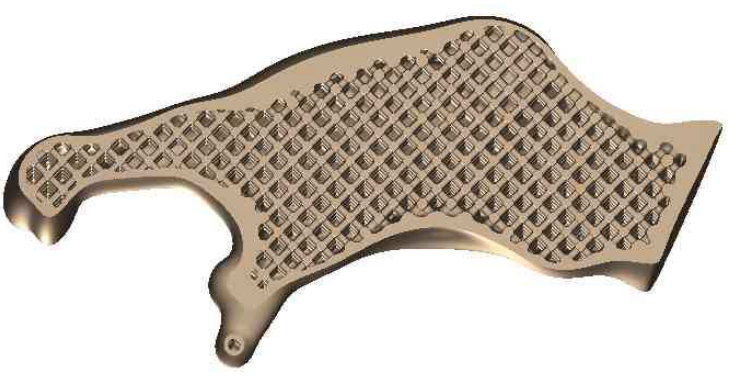

(c)

Figure 5: Lattice scaffold design for a jaw bone: (a) the given shape of a jaw bone; (b) truncation of the microstructure by intersecting it with the bone model; (c) the resulting scaffold as union of the truncated microstructure with the object shell. 


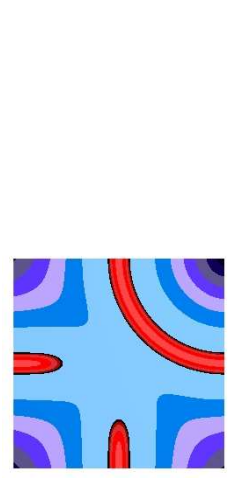

(a)

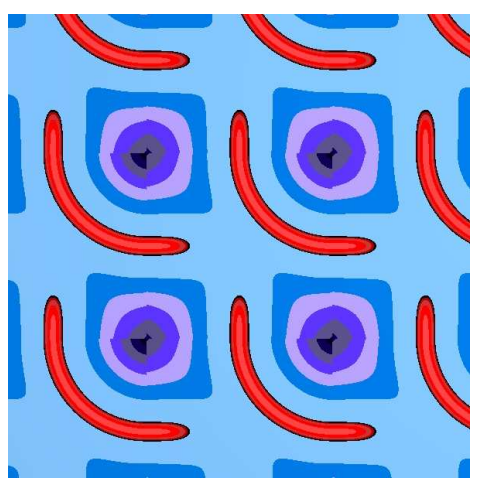

(b)

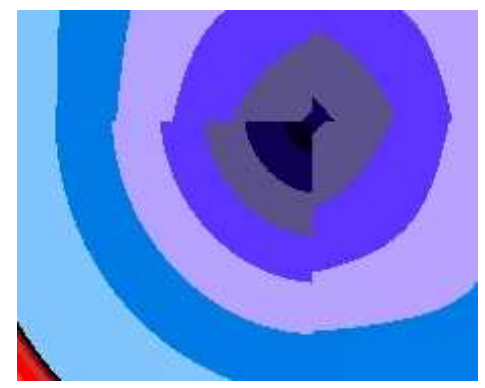

(c)

Figure 6: Discontinuity for geometric connectivity: (a) contour plot of the unit cell from figure 9, (b) contour plot of the replication, (c) zoom on the area of $C^{0}$ discontinuity.

\subsection{Modelling cellular microstructures}

The known approach to modelling microstructures based on the cell (truss) replication is similar to texture tiling. The modelling process consists of two steps: first, a geometric model of the base object (so-called unit cell) is prepared, and secondly, the unit cell is replicated in space to form the cellular microstructure. In the case of BRep and voxel models, a finite set of unit cell copies are combined inside a finite bounding box. To obtain an FRep model of an infinite cellular structure, we apply a periodic function defining a space mapping to the FRep model of the unit cell geometry.

Given a geometric object defined by the continuous real function $f(x, y, z)$ on the interval $I=\left(x_{\min } \leq x \leq\right.$ $\left.x_{\max }, y_{\min } \leq y \leq y_{\max }, z_{\min } \leq z \leq z_{\max }\right)$ and the periodic replication function $g(t)$ such as $g(t) \in[0,1] \forall t$, the cellular model is defined by the following equation:

$$
\begin{array}{r}
r(x, y, z)=f\left(x_{\min }+g(x) *\left(x_{\max }-x_{\min }\right),\right. \\
y_{\min }+g(y) *\left(y_{\max }-y_{\min }\right), \\
\left.z_{\text {min }}+g(z) *\left(z_{\max }-z_{\min }\right)\right)
\end{array}
$$

The object defined by the function $f$ on $I$ is called

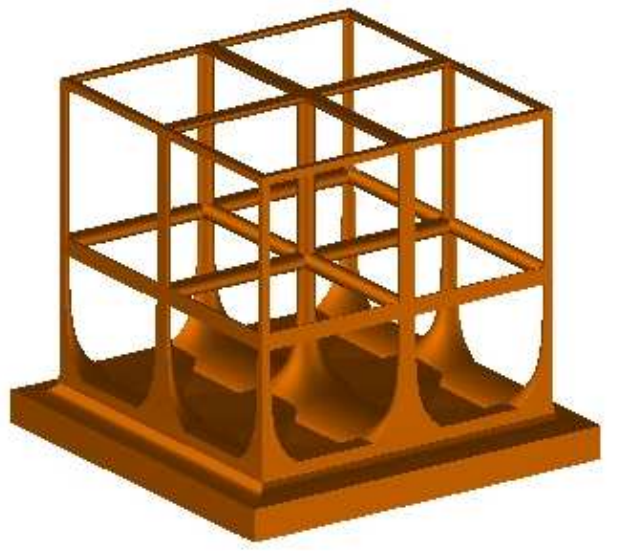

(a)

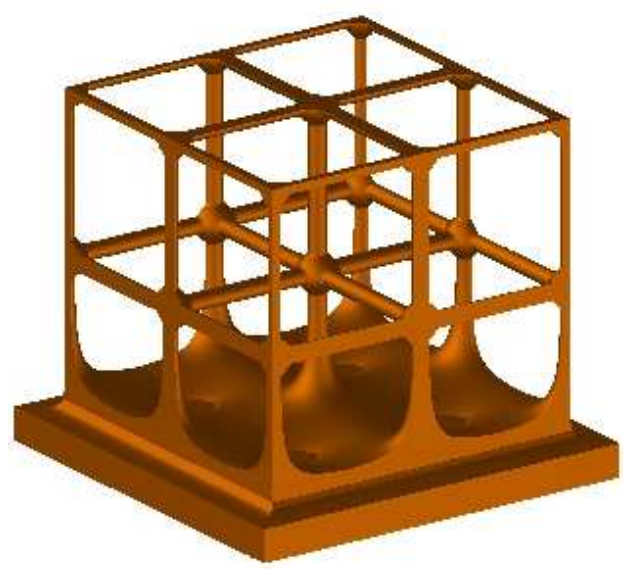

(b)

Figure 7: Application of different types of replication functions for blending operation of the object with cellular structures: (a) Sawtooth wave, (b) Triangle wave. 


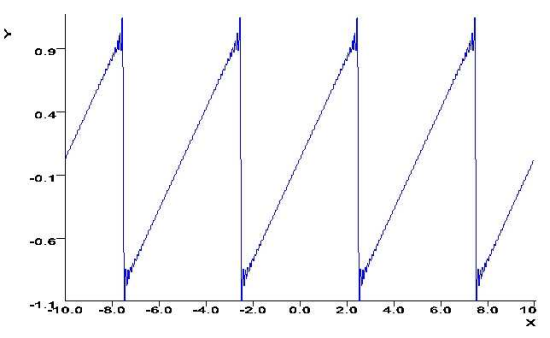

(a)

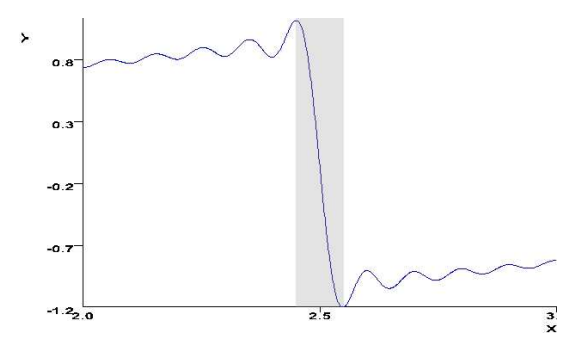

(b)

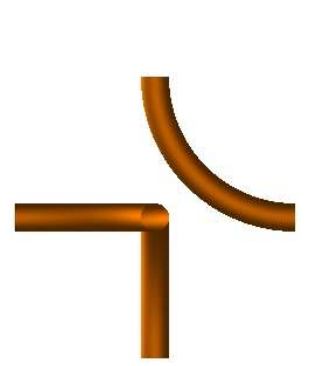

(c)

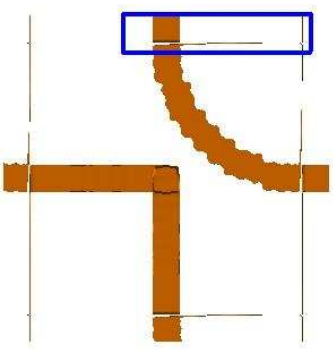

(d)

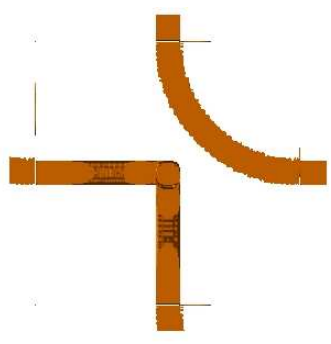

(f)
Figure 8: Unit cell replication using the Fourier sawtooth wave: (a) Fourier sawtooth function with 50 harmonics; (b) zoom to the most problematic area of the Fourier sawtooth (marked in grey) creating artefacts; (c) unit cell geometry; (d) unit cell replication with 20 harmonics; (e) zoom to the artefacts in the neighbourhood of the unit cell faces; (f) unit cell replication with 50 harmonics.

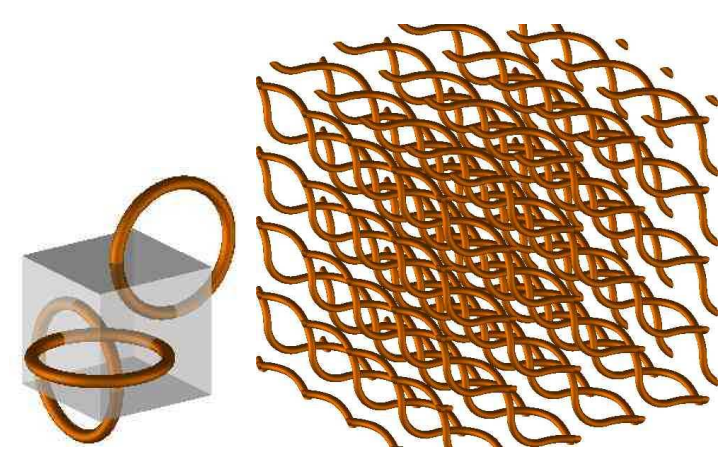

(b)

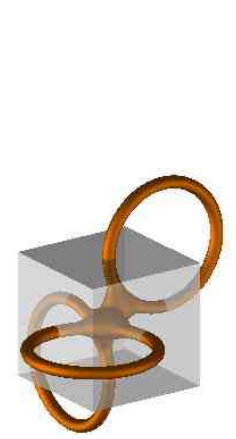

(c)

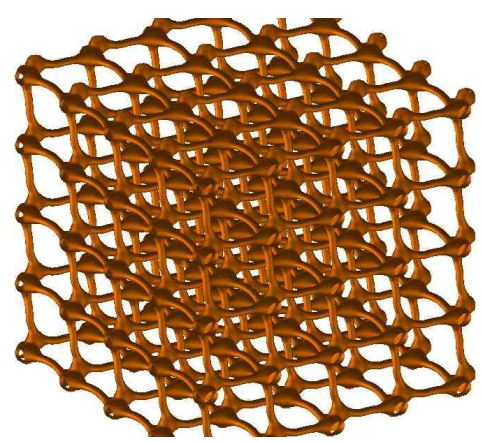

(d)
Figure 9: Cellular microstructures: (a) unit cell, (b) replication of the unit cell, (c) unit cell with added blending, (d) replication of the unit cell with blending. 
a unit cell. The function for the unit cell has to provide the connectivity necessary for the properties of the cellular structure. We distinguish two different types of connectivity. First is geometric connectivity, where the criterion is:

$$
\begin{array}{ll}
f\left(x_{\text {min }}, y, z\right)=0, \text { if } f\left(x_{\text {max }}, y, z\right)=0 & \forall y, z \in I \\
f\left(x, y_{\text {min }}, z\right)=0, \text { if } f\left(x, y_{\text {max }}, z\right)=0 & \forall x, z \in I \\
f\left(x, y, z_{\text {min }}\right)=0, \text { if } f\left(x, y, z_{\text {max }}\right)=0 & \forall x, y \in I
\end{array}
$$

This means the object's boundary curves at the opposite faces of the unit cell bounding box have to be congruent. This property ensures that the replicated cells of the overal cellular structure are connected to each other at these curves.

The second type is full connectivity, where the criterion is:

$$
\begin{array}{ll}
f\left(x_{\min }, y, z\right)=f\left(x_{\max }, y, z\right) & \forall y, z \in I \\
f\left(x, y_{\min }, z\right)=f\left(x, y_{\max }, z\right) & \forall x, z \in I \\
f\left(x, y, z_{\text {min }}\right)=f\left(x, y, z_{\max }\right) & \forall x, y \in I
\end{array}
$$

Such a property ensures the defining function continuity for the entire cellular structure.

We select the sawtooth function as the basic replication function:

$$
g(t)=\frac{1}{2}+\left(\frac{t}{a}-\text { floor }\left(\frac{t}{a}+\frac{1}{2}\right)\right)
$$

Because of the nature of the sawtooth function, the resulting cellular model has geometric discontinuity on the faces of the cells in case the unit cell has no connectivity property. In case of the geometric connectivity, we obtain geometric continuity of the entire model, however the resulting function can be $C^{0}$-discontinuous on the faces of each cell (see Fig. 6). In case of the full unit cell connectivity we have $C^{0}$-continuous function in the entire domain, but $C^{1}$-discontinuity can appear on the cell faces and cause surface artefacts in blending and other operations. However, we can obtain $C^{1}$ continuous function for the unit cell geometry symmetric in respect to the planes parallel to the unit cell faces and passing through its centre. In this case we should use a different replication function, the triangle wave instead of the sawtooth wave:

$$
g(t)=1+\frac{2}{\pi} \arcsin \left[\sin \left(\pi\left(t-\frac{1}{2}\right)\right]\right.
$$

A similar approach is presented in [19]. For triangle wave we obtain $C^{1}$-continuous function, but restrict unit cell to be symmetrical as described above (see Fig

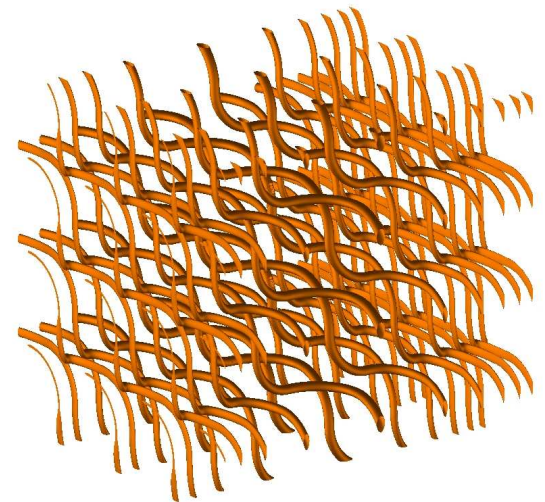

(a)

Figure 10: Frequency variation for cellular structure.

7). In general, the differential properties of the regular lattice models described earlier are better than those of the cellular structures. On the other hand, regular lattices are quite limited in their geometry and require new formulae for each geometry type.

We can apply a Fourier series representing the sawtooth wave instead of the standard sawtooth function:

$$
g(t)=\frac{1}{2}-\frac{1}{\pi} \sum_{k=1}^{n} \frac{\sin (2 \pi k a t)}{k}
$$

Here $n$ is the number of harmonics in the series. The Fourier series function allows to have continuity of $C^{1}$ and higher. However, in this case we have to select a very big number of harmonics to have a good approximation of the sawtooth function. Fig. 8 illustrates the fundamental problems with employing the Fourier sawtooth wave for the replication. In Fig. 8d and Fig. 8f it can be seen that even 50 harmonics are not enough for a good approximation. Moreover, the replication based on the Fourier series adds artefacts (projectiontype copies of the unit cell geometry) to the neighbourhood of the cell faces (clearly visible in Fig. 8e), which is inappropriate in most cases and needs further research for improvement.

Fig. 9 illustrates the steps of the cellular microstructure design. First, an FRep model of the unit cell has to be defined (Figs. 9a, c). Then, the replication function is applied to the part of the model placed inside the unit cube (shown in grey). Note that the truncation of the unit cell geometry by the unit cube occurs automatically due to the sawtooth function construction. The resulting cellular microstructure (Figs. 9b, d) is infinite in space and can be combined with an arbitrary geometric object similar to the case of lattices. In case the mi- 
crostructure is not manufacturable as in Fig. 9b (it will not hold together after the support material is removed) some modifications to the unit cell geometry have to be made such as bounded blending [22] between its disconnected components (Fig. 9c).

We can apply additional transformation for replication function to obtain variation of the shape of the resulting cellular structure. For example, we can have frequency in the sawtooth function be dependent on the coordinate value and thus vary the frequency (see Fig. $10)$.

\section{Modelling irregular microstructures}

Irregular microstructures such as porous media need an approach different from the one suitable for modelling the regular rectangular lattices. As we could see from the survey of existing works, interconnected spherical pores with pseudo-random variations of sizes and positions are typically used in the porous structure analysis. We can replicate the basic pore shape with a periodic space mapping similar to cellular structures, but the overall microstructure connectivity is not an issue in this case. We propose the following modelling procedure:

1. select a basic pore shape such as a sphere or an ellipsoid;

2. replicate the basic pore in space with variable parameters, if necessary;

3. introduce pseudo-random variations of the pores positions and sizes;

4. subtract the pores from the given initial shape.

The proposed procedure is illustrated by Figs. 11 ac. A basic pore with the defining function $F_{p}$ can be replicated in space (Fig. 11a) by the following space mapping:

$$
\begin{aligned}
& x^{\prime}=p_{x} \sin \left(q_{x} x\right) \\
& y^{\prime}=p_{y} \sin \left(q_{y} x\right) \\
& z^{\prime}=p_{z} \sin \left(q_{z} x\right) \\
& F_{r}=F_{p}\left(x^{\prime}, y^{\prime}, z^{\prime}\right)
\end{aligned}
$$

where $p_{x}>0$ specifies a linear scaling for the $x$ coordinate and $q_{x}>0$ controls the frequency of the basic pore replication along the $x$-axis. The replicated pores make actual cavities in the initial shape (sphere in Fig. 11) with the defining function $F$ through the settheoretic subtraction:

$$
F_{m}(x, y, z)=F \backslash_{\alpha} F_{r}
$$

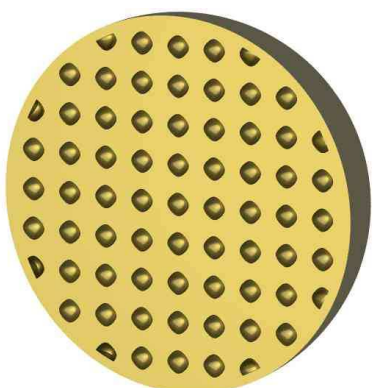

(a)

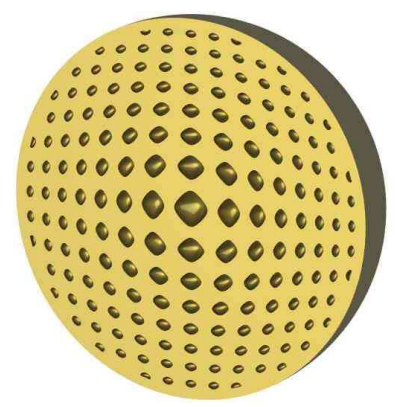

(b)

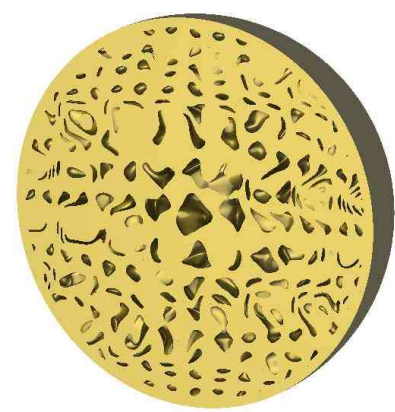

(c)

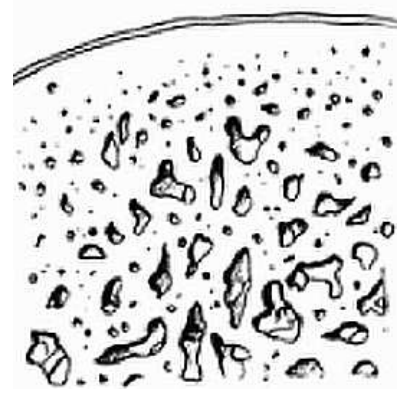

(d)

Figure 11: Function-based porous media modelling: (a) the basic pore replicated in space and subtracted from the initial shape; (b) the pore sizes made decreasing with the distance to the surface; (c) the pseudorandom variations of pores' parameters; (d) the cross section of a sample bone for comparison. 
Similar to the case of lattices, a pore size can be made variable depending on the distance to the initial surface (Fig. 11b). Finally, the pseudo-random deformations of pores can be introduced by adding a solid noise function [23] to any of the model parameters. In the example of Fig. 11c, the Gardner noise [23] with different parameters was added to the values of scale $\mathrm{p}$, frequency $\mathrm{q}$, and the basic pore function $F_{p}$. The solid noise parameters were selected to generate the pore shapes similar to those in the sample bone cross-section (Fig. 11d). More sophisticated methods are definitely required to ensure the model adequacy to the samples of natural porous media. For example, the model parameters can undergo non-linear fitting to satisfy some criteria such as the minimal difference between the model porosity and the real object porosity in the case of irregular structures.

\section{Fitting microstructures}

We need to compare generated microstructures with those obtained by means of magnetic resonance imaging and other measurements of natural or artificial objects. This is useful for both the model adequacy analysis and the parameters estimation of the newly measured objects.

For each type of microstructure (lattice, cellular, nonregular) and each domain of application, it is possible to design a generic parameterized template model. This model can later be tuned to fit the acquired data. For example, a model for the cellular microstructure illustrated in Fig. 9b with the union of three torii as a unit cell, can be adapted to various sets of acquired data that would correspond to a similar structure but with different parameter values (bigger internal radius for the torii, different center locations, etc). In this section, we investigate algorithms for fitting a parameterized microstructure model to a set of three dimensional points positioned on or near the surface of a microstructure object.

Parameters estimation is done by minimizing the residual error of the model against the measured data points. The residual error of the model can be simply computed as the sum of the squared values of the model defining function at each sample. The residual error to be minimized is a complicated non-linear function of the model parameters, and applying standard methods such as the Levenberg-Marquardt algorithm [24, 25] can easily fail (i.e., converge to a local minimum) unless starting from a good approximation of the parameters. To solve this problem, we use a combination of a stochastic global optimization algorithm known as sim- ulated annealing (SA) $[26,27,28]$ with the LevenbergMarquardt algorithm.

\subsection{Algorithm}

Our algorithm optimizes the parameters $p$ of a microstructure model by minimizing the objective (fitness) function $g$ defined using the microstructure template model function $f$ and the point set $p s$ as follows:

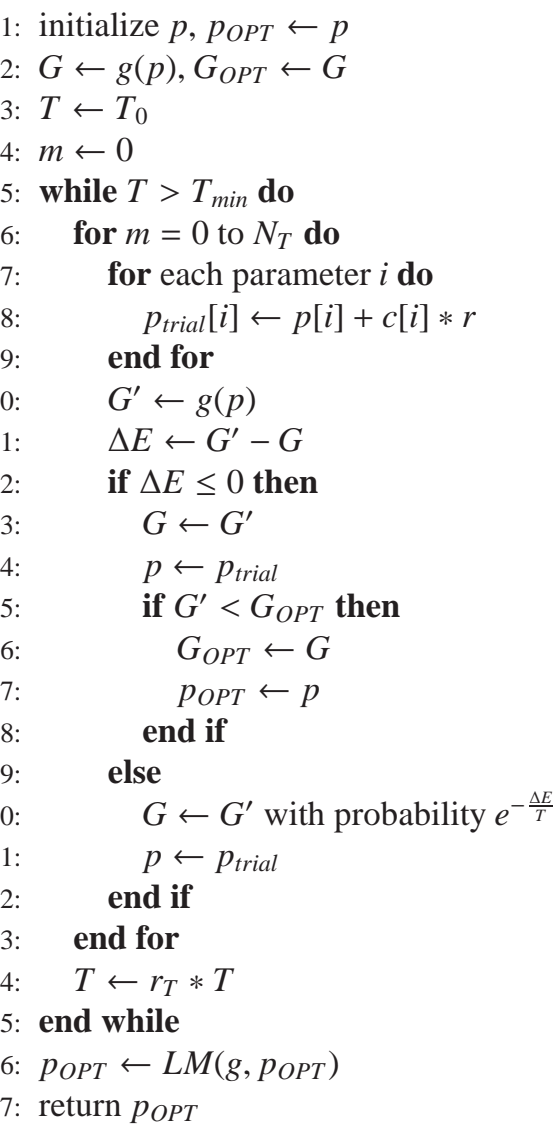

The lines 1 to 4 initialize different variables of the algorithm: $T$ is the variable that simulates the temperature of the system in the SA algorithm and decreases with each iteration, $G$ keeps track of the value of the previous objective function evaluation and $m$ defines the number of steps before a reduction of the temperature. The combination of the initial temperature $T_{0}$, the reduction factor $r_{T}$ and $N_{T}$ forms the cooling schedule of the SA algorithm. In our experiments, we used the following values: $T_{0}=1000, r_{T}=0.85$ and $N_{T}=200$.

The main part of the algorithm is the loop in the lines 5 to 25 . In the lines 7 to 9 , a new vector of parameters is generated by adding to each parameter a random value in the range $[-1,1]$. The variable $c$ is used to limit the search space in each direction. If a parameter is outside 
of the search space after its modification, the step 8 is iterated one more time.

In the lines 12 to 18 , if the new parameter values result in improvement they are always kept. A worst solution can still be accepted with a probability $e^{-\frac{\Delta E}{T}}$ (lines 19 to 22). The best vector of parameters and the best function value found so far are also kept in lines 15 to 18.

After $N_{T}$ function evaluation steps, the temperature of the system is decreased (line 24), making the acceptance of worst solutions less likely. Finally, after the temperature has reached some minimial threshold (other termination criteria can be used as well), the LevenbergMarquardt algorithm is used with the best found solution $p_{O P T}$ as an initial estimation (line 26).

\subsubsection{Choices for the objective function}

The presented algorithm aims to minimize the objective function $g$ defined using the microstructure template model function $f$ and the set of the points scattered on the surface $p s$. The simplest choice for the objective function is to use the least square error: $g(p)=$ $\sum_{i} f^{2}(p s[i], p)$.

A slightly better choice is to use a decreasing exponential: $g(p)=\sum_{i} e^{-\frac{f^{2}(p s[i], p)}{\sigma^{2}}}$, which acts similarly to counting the number of points which are within a distance $\sigma$ to the surface represented by $f=0$. In this case the problem becomes a maximization problem and the signs in the algorithm above should be changed accordingly (lines 12 and 15).

\subsubsection{Choices for the termination criteria}

The termination criteria in the above algorithm is rather simple: it terminates when the system temperature is below the given threshold $T_{\min }$. In our experiments, we used $T_{\min }=10^{-5}$. More sophisticated termination criteria involve monitoring the values of the objective function during the algorithm's iterations. The following criteria was used in our experiments: the loop (lines 5 to 25 ) terminates if $G_{O P T}-G^{\prime}<\epsilon$ and if the difference between $G^{\prime}$ and the last five best values of $G^{\prime}$ are not exceeding $\epsilon$. A value of 100 was used for $\epsilon$ in all our experiments.

\subsection{Experimental results}

We illustrate our algorithm with three examples using two types of regular micro-structures: lattice and cellular.

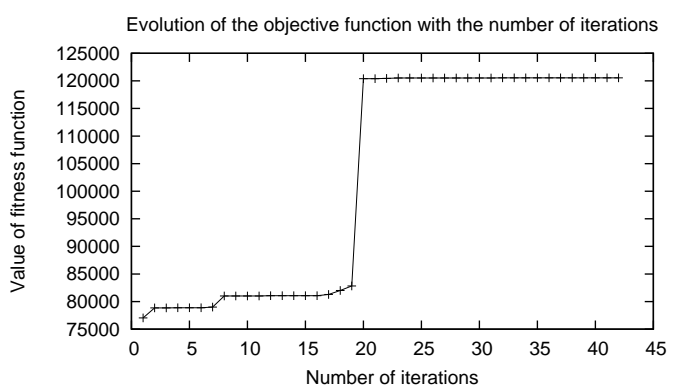

Figure 12: Evolution of the objective function with the number of iterations of the SA algorithm for a regular lattice model.

\subsubsection{Regular lattice}

The first example corresponds to the regular lattice model illustrated in Fig. 4c. Our model has seven parameters corresponding to the scale and frequency of the slabs (see Eq. 4) and the parameters of the blending set operations (see Eq. 8). The frequency and scale of the model vary in space: they are controlled by the distance to the shell which is used to interpolate between two boundary values of the frequency and scale.

A set of 385,535 points scattered on the surface of the object is used as the target for fitting the chosen seven parameters of the model. The decreasing exponential (see section 5.1.1) with the value $\sigma=10^{-3}$ was used as the objective function optimized by the algorithm discussed in section 5.1.

The termination of the main loop occurred after 42 iterations, taking approximately $20 \mathrm{~min}$ on a Sun workstation with $2.8 \mathrm{Ghz}$ Intel Xeon processors. The evolution during the algorithm's iterations of the value of the objective function corresponding to the best solution ( $G_{O P T}$ and $p_{O P T}$ in the algorithm above) is illustrated in Fig. 12. The initial values of the parameters are randomly selected: $5.624,2.581,1.921,3.254,4.743$, 0.941 and 3.541. The final values are: $8.999,3,0.759$, $0.84,0.959,3.103$ and 3.103 corresponding respectively to the scale and frequency of the slabs ( 2 parameters for the scales and 2 parameters for the frequencies) and the three parameters controlling the blending union (see Eq. 8).

\subsubsection{Cellular microstructure}

The second example used to illustrate our algorithm corresponds to the cellular microstructure in Fig. 9b. A template model with 14 parameters is used and fitted to points scattered on the surface of the object. The parameters consist of the center coordinates of the torii and their radii as well as the scale parameter of the sawtooth 


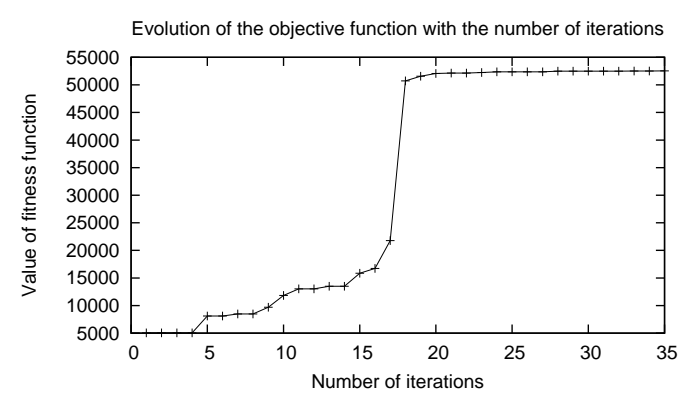

Figure 13: Evolution of the objective function with the number of iterations of the SA algorithm for the cellular microstructure made of three torii.

function (see Eq. 13).

The data set is made of 53,400 points scattered on the surface of the object. The same settings as in the previous example were used for the algorithm. The termination of the main loop occurred after 35 iterations in approximately $10 \mathrm{~min}$. The evolution of the objective function corresponding to the best solution is illustrated in Fig. 13. The initial values for the parameters are randomly chosen: 5.187, 0.258, 0.3778, $0.581,0.479,-0.985,0.607,-0.821,-0.016,-1.126$, $-1.248,-0.331,-0.668$, and -0.396 . The final values are: 5.003, 0.999, 0.999, 0.999, 0.1, $-0.999,0.999,0,0$, $-0.999,-0.999,0.999,0$, and 0.999 corresponding respectively to the scale parameter of the sawtooth function, the radii of the torii and the coordinates of each torus center.

We measure the approximation error at each point by the square of the microstructure function value at that point. The best found parameters are used in the model. Fig. 14 illustrates the point-set on the surface where each point is coloured according to the approximation error.

The model for this cellular microstructure can be extended by replacing the union operation between the unit cells by a blending union as illustrated in Figs. 9c, d. If the blend described in Eq. 8 is used, then three more parameters are added to the precedent model. If the bounded blend operation described in [22] is used, then four additional parameters are needed. We need also to add at least one more parameter for the function localizing the blend. We found in our experiments that fitting the parameters corresponding to the blending operation was a difficult task. Fig. 15 plots the least square error of the model for different values of the coefficient $a_{0}$ governing the blend and the radius $r$ of the sphere used to localize the blend. It can be seen that the pa-

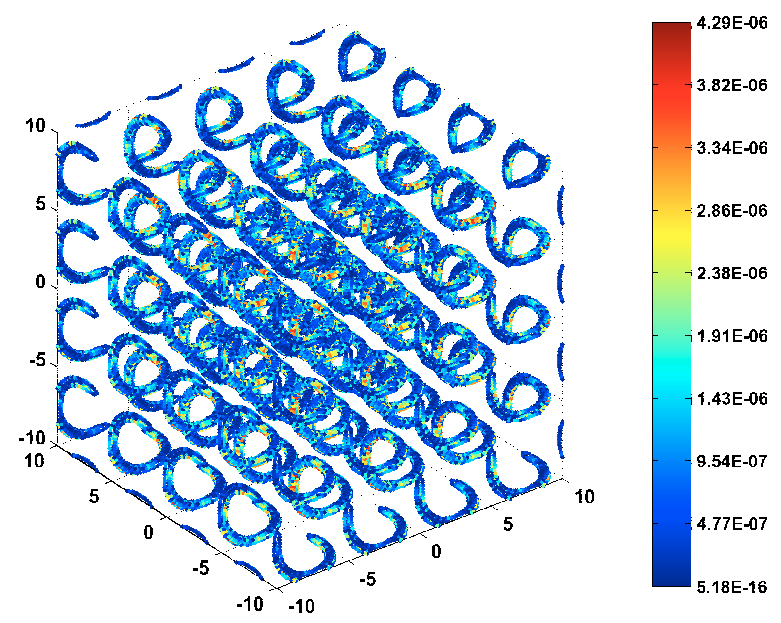

Figure 14: Point-set on the surface coloured according to the approximation error $\left(f^{2}\right)$ of the optimized model for the cellular microstructure.

rameter space is extremly flat making the optimization difficult.

Evolution of the objective function value with the number of iterations is illustrated in Fig. 16. The algorithm converged to a reasonable result after 35 iterations in approximately $20 \mathrm{~min}$. The initial values for the parameters are randomly chosen: 8.520, 0.314, $0.388,-0.482,-1.349,-0.0450,1.192,-0.773,0.082$, $-0.017,1.065,-0.462,0.562$ and 0.223 . The final values are: 5.001, 0.995, 0.1, $-0.997,0.995,7 e-05$, $1 e-5,-0.995,-0.996,0.996,1 e-05,0.996,0.795$, 0.081 corresponding respectively to the scale parameter of the sawtooth function, the torii radius (2 parameters), the coordinates of the torii centers ( 9 parameters) and finally the parameters for the bounded blending union (last 3 parameters).

For the first two examples, it was possible to use a faster cooling schedule (by decreasing $N_{T}$ or $T_{0}$ ) while getting similar results. However for this third example, a slow cooling schedule was needed to reach an acceptable result.

\subsection{Discussion}

Minimization (or maximization) of non-linear objective functions is in general a difficult task if we do not know apriori a good starting point. The algorithm proposed here has provided acceptable results in our experiments. This type of algorithm is however expensive as it requires a significant number of evaluations of the objective function. For our domain of application, the objective function is defined itself as the sum 


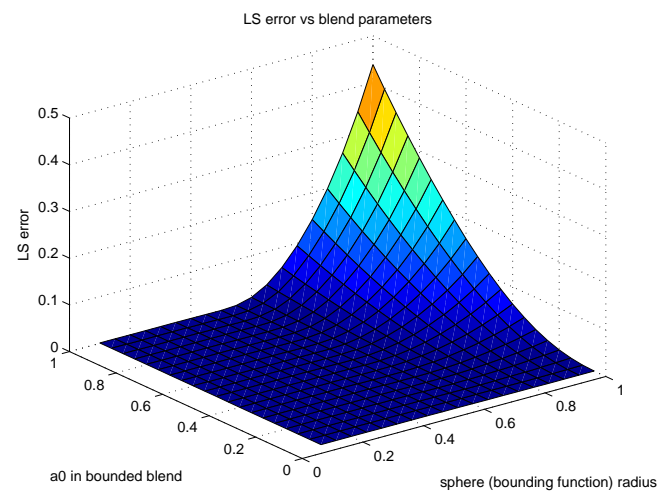

Figure 15: Variation of the least square error with two of the parameters used in controlling the blend between the unit cells of the cellular micro-structure.

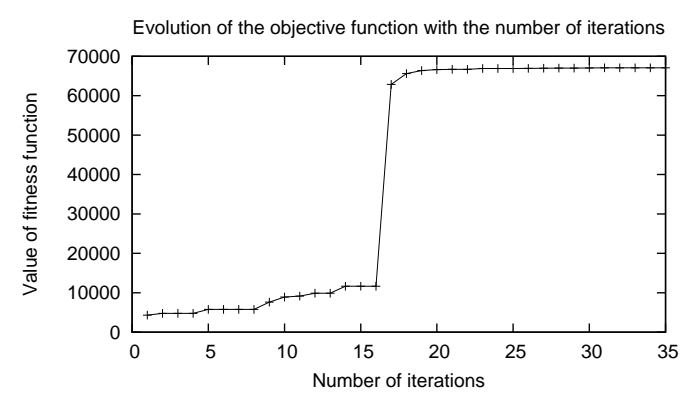

Figure 16: Evolution of the objective function with the number of iterations of the SA algorithm for the cellular microstructure using blend. over a number of points of a function of space coordinates. The objective function is consequently already expensive to compute (in the examples described above the sums were over approximately 400,000 and 60,000 points). The reduction of the size of the input point set in a pre-processing step is one possible approach to decrease the time spent for optimization. This can be done using clustering (for example, k-means clustering) and replacing each cluster by its closest neighbour in the original point-set (using, for example, a kd-tree). We have not explored this possibility yet.

In the evaluation of the objective function, each computation in the sum is independent, so it is easy to distribute the total computation using several threads on a multi-core (or multi-cpu) computer and this can provide an improvement of the running time.

The cooling schedule used is a reduction by a constant factor $r_{T}$ (0.85 in our experiments) of the previous temperature at every $N_{T}$ time step (200 in our experiments), starting from an initial temperature $T_{0}$ (1000 in our experiments). The cooling schedule is an important part of the algorithm: if it is too slow, it will result in a long running time for the algorithm; if it is too fast, the algorithm may converge to a local optimum only. A lower starting temperature (100) and a smaller $N_{T}$ worked for the first and second examples described above but failed for the last one. These parameters are likely to be dependent on the model, and we gave values that had worked for the examples above. They can probably be better estimated by an initial sampling of the objective function but this is a part of a future work.

\section{Direct rendering and fabrication}

Application areas of microstructure modelling such as composite material design and biomedical tissue engineering require both model rendering and manufacturing procedures. Typically a function-based model has to be converted to some auxiliary representations for subsequent rendering using modern graphics hardware and then for manufacturing on rapid prototyping or 3D printing equipment. The conversion to BRep involves an isosurface polygonization (tessellation) while the voxelization is needed to produce a voxel array representation. The disadvantages of both these auxiliary representations in the case of microstructure modelling were discussed in Introduction. A more promising approach can be called direct rendering and fabrication.

Direct rendering of isosurfaces can be done with accelerated ray-tracing/ray-casting. In [29] it was shown that using of GPU allows direct rendering of relatively complex function-based models with interactive rates 


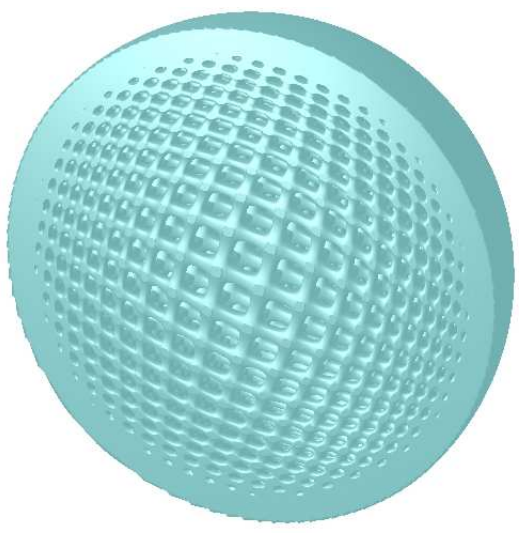

(a)

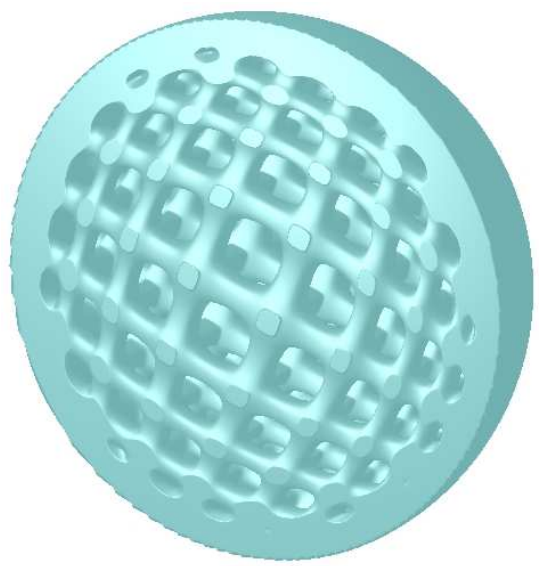

(b)

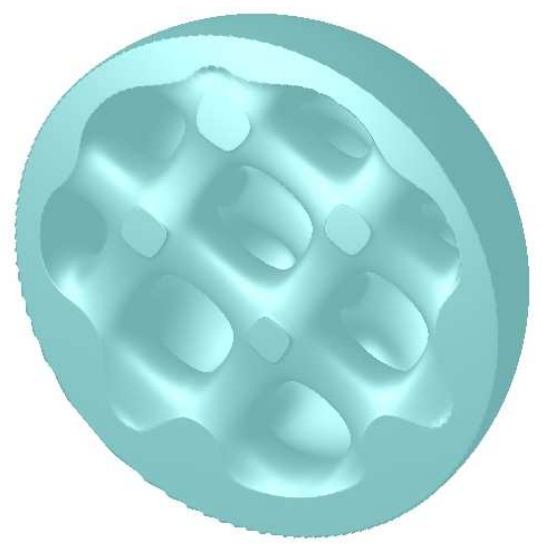

(c)

Figure 17: Near real-time ray-casting of a spherical thin shell with the internal regular lattice of different density; GPU rendering rate remains about 20 frames per second independent of the microstructure complexity (image size $512 * 512$ )

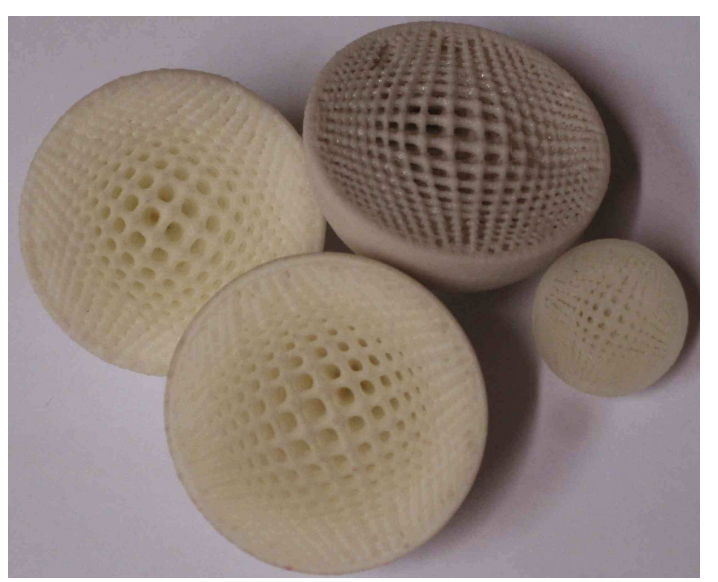

Figure 18: The model of Fig. 4c fabricated in several forms - from right to left: polyamide material using a 3D Systems Sinterstation HiQ, impregnated plaster using a ZCorp 3D printer and FDM deposited plastic filament using a Stratasys Dimension 3D Printer.

on modern graphics hardware (PC with NVidia 9600 graphics card). An example of ray-casting of the sphere with microstructures is shown in Fig. 17. As the complexity of the function does not depend on the density of microstructures, the rendering time is almost the same for lattices with different densities on the given graphics hardware.

Several of the FRep based microstructure models described above have been fabricated using a variety of digital fabrication machines and materials. For example, Fig. 18 shows a fabricated model of a lattice parametrised by the distance to the external surface (Fig. $4 \mathrm{c})$. The models were first polygonized and then output as STL files. The STL files were imported into the various software packages for driving the machine where they were checked for surface defects and then machine paths were generated based on layered slices of the STL and manufacturing constraints. Many of the models must also go through post processing to remove support structure and/or add additional strength. During the manufacturing process, the STL file format created many issues for the slicing, path planning and fabrication of some of the fine microstructures.

A much better approach is to directly fabricate the FRep model without poor intermediate formats such as STL. This has been previously proposed in [30] and is an active ongoing focus of our research. One possibility is to produce a raster image for each layer of manufacturing at the machine resolution, which is an acceptable input for some existing machines. Thus the microstructure could be procedurally defined on a voxel-grid that corresponds directly to the layer thickness and to the 


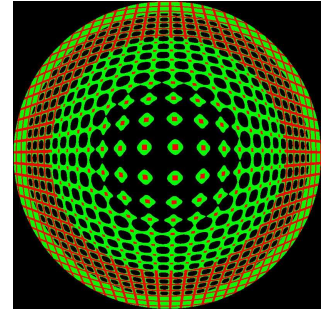

(a)

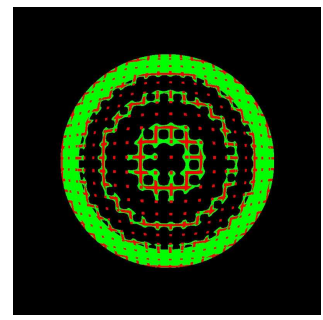

(c)

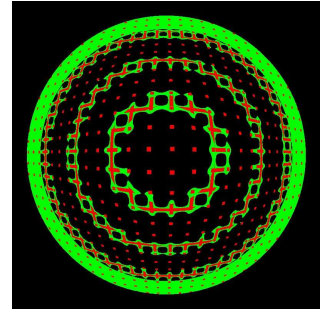

(b)

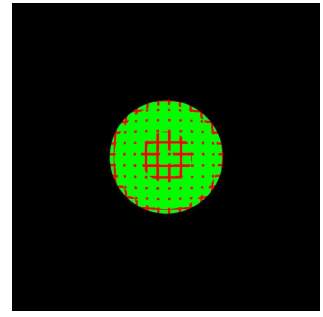

(d)
Figure 19: Raster image layers for direct fabrication of a multimaterial version of the object of Fig. 4c: microstructure rods are made of one material and another material is added by blending

pixel spacing of the printing process. Fig. 19 shows several raster image layers of a multi-material version of the object in Fig. 4c. It is quite easy to distinguish using the FRep model the lattice rods and the material added by the blending union operation. In this model, the rods are made of one material and another material is added by blending.

Another choice is to directly control the digital fabrication process including the tool motions and material deposition. However, the obstacles presented by the proprietary nature of most digital fabrication technologies, such as access to machine protocols and control commands, require open hardware systems such as the Fab@Home or RepRap 3D printers. Unfortunately, the resolution of these free and open source systems is currently not competitive with high end commercial machines necessary for fine resolution microstructures.

\section{Discussion and conclusions}

We have proposed several approaches that use periodic functions in different ways for modelling both regular and irregular microstructures within the FRep framework. In the case of lattices, these functions serve to directly define the point membership by analysing the sign of the function. In the case of cellular structures and porous media, the periodic functions are used for space mapping (coordinates transformations) such that some basic shape can be infinitely replicated in space. A simple spherical pore was tested; however, any porous shape can be involved without changes in the rest of the generation procedure.

One could observe that the differential properties of the regular lattice models are better than those of the cellular structures. The provided $C^{1}$-continuity of the functions for lattices allow for blending operations without any surface artefacts. On the other hand, the model for cellular structures is a more general approach, supporting practically arbitrary geometry of the unit cell, which is very useful for interactive modelling.

The proposed models are extremely compact (all examples in this paper can be implemented in 10-20 lines of HyperFun [20] or in 30-40 lines of C language), while providing precise and spatially coherent models. Compared to methods using BRep, FRep parametrization provides more robust and dynamic control, including parameter-dependent changes in object's topology. The FRep models of microstructures can be used as arguments for further set-theoretic, blending, offsetting, and other geometric operations. These models can be directly rendered using ray-tracing with interactive rates in the case of the GPU implementation.

Manual creation of models for each particular instance of the microstructure of the given type is tedious work. We present a method for the automatic estimation of parameters of the given template model by fitting it to scanned or otherwise measured surface points. Our experiments showed promising results, however, such structures as porous bones and other irregular structures need more sophisticated approaches.

The direct fabrication of microstructures without generating any auxiliary representations is one of the immediate subjects for our research and development. As the model strength can be one of the design criteria, the strength analysis will be an area of our future research, based on the heterogeneous objects discretization and finite element meshes generation reported in [31].

The primary subject of this work was modelling volumetric microstructures and it will be a subject of future research to find out if this approach can be applied to material microstructures such as grains, grain boundaries and secondary phases such as nanofibers. Finally, we intend to develop function-based models for other types of volumetric microstructures such as octahedral lattices, natural branching and organic structures.

\section{Acknowledgment}

The authors would like to thank Denis Kravtsov for the FRep based model of the jaw bone. We are also 
thankful for the support of Arnaldo Luis Lixandrao Filho, Paulo Inforcatti Neto, Tatiana Al-Chueyr and Jorge Vicente Lopes da Silva at the Centro de Tecnologia da Informacao - CTI in Brasil, who helped make this work possible by digitally fabricating many models and providing medical data.

\section{References}

[1] W. Sun, B. Starly, J. Nam, A. Darling, Bio-cad modeling and its applications in computer-aided tissue engineering, ComputerAided Design 37 (11) (2005) 1097-1114

[2] X. Y. Kou, S. T. Tan, Heterogeneous object design: an integrated cax perspective, in: Heterogeneous objects modelling and applications: collection of papers on foundations and practice, Springer-Verlag, Berlin, Heidelberg, 2008, pp. 42-59.

[3] C. K. Chua, W. Y. Yeong, K. F. Leong, Rapid prototyping in tissue engineering: a state-of-the-art report, in: Proc. 2nd Int. Conf. on Advanced Research in Virtual and Rapid Prototyping, 2005, pp. 19-27.

[4] M. W. Naing, C. K. Chua, K. F. Leong, Y. Wang, Fabrication of customised scaffolds using computer-aided design and rapid prototyping techniques, Rapid Prototyping Journal 11 (4) (2005) 249-259.

[5] H. Wang, Y. Chen, D. W. Rosen, A hybrid geometric modeling method for large scale conformal cellular structures, ASME Conference Proceedings 2005 (47403) (2005) 421-427.

[6] Y. Chen, 3d texture mapping for rapid manufacturing, Computer-Aided Design \& Applications 4 (6) (2008) 761-771.

[7] V. Gervasi, A. Schneider, J. Rocholl, Geometry and procedure for benchmarking SFF and hybrid fabrication process resolution, Rapid Prototyping Journal 11 (1) (2005) 4-8.

[8] S. Hanna, Inductive machine learning of optimal modular structures: Estimating solutions using support vector machines, $\mathrm{Ar}$ tificial Intelligence for Engineering Design, Analysis and Manufacturing 21 (04) (2007) 351-366.

[9] E. Vergés, D. Ayala, S. Grau, D. Tost, 3d reconstruction and quantification of porous structures, Computers \& Graphics 32 (4) (2008) 438-444.

[10] Y. Holdstein, A. Fischer, L. Podshivalov, P. Bar-Yoseph, Volumetric texture synthesis of bone micro-structure as a base for scaffold design, in: Shape Modeling and Applications, 2009. SMI 2009. IEEE International Conference on, IEEE Press, 2009, pp. 81-88.

[11] P. Lal, W. Sun, Computer modeling approach for microspherepacked bone scaffold, Computer-Aided Design 36 (5) (2004) 487-497.

[12] C. Schroeder, W. C. Regli, A. Shokoufandeh, W. Sun, Computer-aided design of porous artifacts, Computer-Aided Design 37 (3) (2005) 339-353.

[13] H. N. Chow, S. T. Tan, W. S. Sze, Layered modelling of porous structures with voronoi diagrams, Computer-Aided Design \& Applications 4 (1-4) (2007) 321-330.

[14] J. Bloomenthal et al. (Ed.), Introduction to Implicit Surfaces, Morgan Kaufmann Publishers Inc., San Francisco, CA, USA, 1997.

[15] A. Pasko, V. Adzhiev, A. Sourin, V. Savchenko, Function representation in geometric modeling: concepts, implementation and applications., The Visual Computer 11 (8) (1995) 429-446.

[16] V. L. Rvachev, Methods of logic algebra in mathematical physics, Kiev Izdatel Naukova Dumka

[17] V. Shapiro, Theory of r-functions and applications: A primer,
Tech. Rep. "Technical Report CPA88-3", Cornell University (1988).

[18] J. Parulek, M. Sramek, I. Zahradnik, Geomcell, in: N. Magnenat-Thalmann, J. J. J. Zhang, D. D. D. Feng (Eds.), Recent Advances in the 3D Physiological Human, Springer London, 2009, pp. 21-36.

[19] N. Stolte, Infinite implicit replication: Case study for voxelizing and representing cyclical parametric surfaces implicitly, in: Proceedings of the Shape Modeling International 2002 (SMI'02), IEEE Computer Society, 2002, pp. 105-110.

[20] V. Adzhiev, R. Cartwright, E. Fausett, A. Ossipov, A. Pasko, V. Savchenko, HyperFun project: a framework for collaborative multidimensional F-Rep modelling, in: Proc Implicit Surfaces '99, Eurographics/ACM SIGGRAPH Workshop, J. Hughes and C. Schlick (Eds.), 1999, pp. 59-69.

[21] V. Shapiro, Semi-analytic geometry with R-functions, Acta Numerica 16 (2007) 239-303.

[22] G. Pasko, A. Pasko, T. Kunii, Bounded blending for functionbased shape modeling, IEEE Computer Graphics and Applications 25 (2) (2005) 36-45.

[23] G. Gardner, Simulation of natural scenes using textured quadric surfaces, SIGGRAPH Computer Graphics 18 (3) (1984) 11-20.

[24] K. Levenberg, A method for the solution of certain non-linear problems in least squares, The Quarterly of Applied Mathematics (1944) 164-168.

[25] D. Marquardt, An algorithm for least-squares estimation of nonlinear parameters, SIAM Journal on Applied Mathematics 11 (1963) 431-441.

[26] N. Metropolis, A. W. Rosenbluth, M. N. Rosenbluth, A. H. Teller, E. Teller, Equations of state calculations by fast computing machines, Journal of Chemical Physics 21 (6) (1953) 1087 1092.

[27] S. Kirkpatrick, C. D. Gelatt, M. P. Vecchi, Optimization by simulated annealing, Science 220 (4598) (1983) 671-680.

[28] A. Corana, M. Marchesi, C. Martini, S. Ridella, Minimizing multimodal functions of continuous variables with the "simulated annealing" algorithm, ACM Trans. Math. Softw. 13 (3) (1987) 262-280. doi:http://doi.acm.org/10.1145/29380.29864.

[29] O. Fryazinov, A. Pasko, Interactive ray shading of FRep objects, in: WSCG' 2008, Communications Papers proceedings, 2008, pp. $145-152$

[30] T. Vilbrandt, E. Malone, H. Lipson, A. Pasko, Universal desktop fabrication, in: Heterogeneous objects modelling and applications: collection of papers on foundations and practice, Springer-Verlag, Berlin, Heidelberg, 2008, pp. 259-284.

[31] E. Kartasheva, V. Adzhiev, A. Pasko, O. Fryazinov, V. Gasilov, Surface and volume discretization of functionally based heterogeneous objects, Journal of Computing and Information Science in Engineering 3 (4) (2003) 285-294. 